\title{
A new Prostate Cancer Surrogate Marker-Vimentin3?
}

\author{
Barbara Köditz $^{1 *}$, Andreas Stog ${ }^{1}$, Heike Göbel ${ }^{2}$, Manuel Huerat Arana $^{1}$, Jochen WU Fries ${ }^{1}$, Axel Heidenreich $^{1,3}$, \\ Melanie von Brandenstein ${ }^{1}$
}

${ }^{1}$ Faculity of Medicine and University Hospital of Cologne, Department of Urology, Germany

${ }^{2}$ Faculity of Medicine and University Hospital of Cologne, Insitute of Pathology, Germany

${ }^{3}$ Department of Urology, Medical University Vienna, Austria

*Corresponding author: Barbara Köditz, Faculity of Medicine and University Hospital of Cologne, Department of Urology, Kerpener Straße 62, 50937 Cologne, Germany

\begin{abstract}
Background: Vimentin3 (Vim3) was recently described as a tumor marker for the direct differentiation between benign and malignant kidney tumors. Our group recently reported correlation between Vim3 overexpression and increasing migration of PCa cell lines.

Objectives: Comparing Vim3 serum levels among different PCa grades.

Design Setting and Participants: serum samples obtained. from PCa patients ( $n=314$ ) between 2017 and 2020 were classified according to the grade group system and analysed by ELISA, and Western Blot to measure Vim3 levels. Additionally, Paraffin embedded human PCa samples from radical prostatectomy specimens were stained immunohistochemically for Vim3 ( $\mathrm{n}=10$ ).

Outcome Measurements and Statistical Analysis: Vim3 levels in serum samples show a correlation with increasing aggressiveness of PCas. ANOVA analysis was used.

Results and limitations: the Vim3 protein level increased significantly $(\mathrm{p}<0.001)$ with increasing biological aggressiveness of human PCa specimens from prostate biopsies and/or radical prostatectomy specimens (Gleason $\geq 8$ ). A "grey-zone" value for Vim3 was determined (300-350 ng/ml). Furthermore, Vim3 levels in the Gleason $>7$ group $(3+4)(4+3)$, metastasis, castration resistant and castration responder was analysed. Vim3 levels significantly decrease in the group of ADT responders. Limitations include the retrospective and single centre design.
\end{abstract}

Conclusion: Vim3 could be a new surrogate biomarker for the classification and graduation of PCa, especially for PCa with increasing metastatic potential. Additionally, we hypothesised that Vim3 determination can be usable for ADT respond control.

Patient Summary: Serum Vim3 levels rise with increasing malignancy of the prostate cancer and correlate with the Gleason Score and therefore could be usable

\section{Introduction}

Endothelin-1 initially described as a vasoconstrictor in 1988 [1] has shown to have multifunctional [1-3] in the development of various types of solid tumors [2]. Through the endothelin A receptor (ETAR) it induces a survival pathway leading to cell proliferation, escape from apoptosis, angiogenesis, invasion and metastasis formation $[3,4]$, thus, elevated ET-1 levels in prostate cancer patients can be associated with higher metastatic potential [6] correlating the ET-1 levels with tumor progression [3]. In contrast, the binding to the endothelin B receptor ETBR results in its clearance and cellular apoptosis. In accordance, Nelson, et al. reported a downregulation in aggressive variants of prostate cancers (PCa) [5]. Such down-regulation of ETBR is based on promotor methylation [3]. The authors recently described a clear correlation between ET-1 overexpression and the production of a truncated version of Vimentin, called Vim3, [7] this production is mediated through the activation of ETAR [12]. As a consequence of a greater presence of vim3, cells lose their normal cellular structure, since Vim3 lacks the C-terminal ending of full-length Vimentin and, therefore, its function in the arrangement. of cell organelles and cell anchoring, [4] hence the importance of full-length vimentin. Prostate cancer patients with elevated levels of Vimentin are known to have a higher potential to develop metastases [9] However, due to the use of different commercially available antibodies, directed against either the full version or both versions of Vimentin, results 
of previously performed experiments are questionable [10]. Vim3 overexpression has previously been shown to correlate with increased metastatic potential in breast cancer cells [11] and with increased migration behaviour in prostate cancer cell lines (PC3, LNCap, and DU145) [12]. Currently, the detection of PCa is based on a combination of serum PSA concentration, PSA velocity, digital rectal examination (DRE), multiparametric magnetic resonance imaging (mp-MRI) and prostate biopsy, since the usefulness ofPSA alone as a biological marker detectable in blood and urine for prostate cancer may be limited as its concentration might increase with medications or in cases of inflammation and benign prostate hyperplasia [5]. Therefore, although a high PSA level can be correlated with the presence of prostate cancer; it can also be the result. of benign prostatic hyperplasia, inflammation and medications [6]. Furthermore, PSA is used to monitor PCa patient response to treatment and recurrence. In addition to the PSA, the Gleason score is used to classify PCa based onmicroscopic examination of prostate biopsies and samples from prostatectomies. Previously, 30-36\% of all diagnosed PCa classified by biopsy were upgraded after prostatectomy [7]. The combination of the PSA value and the mp-MRT reduces this discrepancy significantly. However, the question is which CaP has the potential to metastasize and which does not. In this sense, it is necessary to improve differential diagnosis and better distinguish high-risk disease; hence, the need for a specific and reliable surrogate biomarker is of great interest. Therefore, our group is interested in knowing if the serum level of Vim3 correlates with the Gleason score and if it is possible to detect biologically aggressive PCas. Another important question is which patients respond to androgen receptor deprivation therapy (ADT) and which are resistant. Therefore, we separated the group of patients into controls, Gleason $>7 \mathrm{~b}$, with metastases, responders to ADT and non-responders to ADT

\section{Materials and Methods}

\section{Patient Cohort}

The analysed cohort consisted of 294 Biobank (BioMASOTA, University Hospital of Cologne, file reference 12-163) frozen serum samples from PCa patients at the Uro-oncology department of the University hospital of Cologne from 2017 to 2020. The samples were classified into risk groups according to the Gleason score obtained from either the punch biopsy or the radical prostatectomy (RP) sample. Table1. Gleason 6 was grouped as low risk $(n=50)$, Gleason $7(3+4),(4+3)$ as intermediate risk $(n=50)$, and Gleason 8 onwards as high risk $(=50)$. The predominant inclusion criteria for the low and intermediate categories were absence of metastasis, prior androgen deprivation therapy (ADT), or recurrence at the time the serum sample was obtained. Three more categories based mainly on the presence of metastasis $(n=50)$, recurrence $(n=44)$, response to ADT $(n=10)$ and do not respond to ADT $(n=10)$ were analysed. For the groups mentioned last, as can be inferred, samples had a Gleason score of 7 or higher. In the metastasis group, more subgroups were made depending on whether the metastasis was identified at the time of PCa diagnosis (pre-PR, $n=20$ ) or at some indistinct time after PR $(n=30)$. Finally, a control group ( $=50$ ) consisted of serum samples from patients from the urology department of the Cologne University Hospital without a history of any type of malignancy, regardless of gender (Table 1). shows the distribution of patients according to risk, metastases, recurrence and response to androgen deprivation therapy groups and their correspondence.

Table 1: Shows the distribution of patients according to risk, metastases, recurrence and response to androgen deprivation therapy groups and their correspondence Gleason score.

\begin{tabular}{|c|c|c|c|c|}
\hline Risk group & Gleason & PSA (ng/ml) & Recurrence & Metastasis \\
\hline Low $(n=50)$ & 6 & $7.4 \pm 1.61$ & No & no \\
\hline $\begin{array}{l}\text { Intermediate } \\
\quad(n=50)\end{array}$ & $\begin{array}{l}7 a(n=25) \\
7 b(n=25)\end{array}$ & $1.49 \pm 3.19$ & No & no \\
\hline high risk $(n=50)$ & $>8$ & $30.4 \pm 7.68$ & No & no \\
\hline $\begin{array}{c}\text { Metastasis }(\mathrm{n}=50) \\
\text { pre RP }(20) \\
\text { after RP (30) }\end{array}$ & $\geq 7 a$ & $\begin{array}{c}77.65 \pm 105.3 \\
181.97 \pm 154.56 \\
14.82 \pm 8.46\end{array}$ & No & $\begin{array}{l}\text { Osseous } \\
\text { and/or visceral }\end{array}$ \\
\hline Recurrence $(n=44)$ & $\geq 7 a$ & $41.3 \pm 5.12$ & No & $\begin{array}{c}\text { PSA relapse } \\
\text { And Materials }\end{array}$ \\
\hline $\begin{array}{l}\text { ADT responder } \\
\quad(\mathrm{n}=10)\end{array}$ & $\geq 7 a$ & $1.22 \pm 0.774$ & No & Yes \\
\hline $\begin{array}{c}\text { ADT non } \\
\text { responder }(n=10)\end{array}$ & $\geq 7 a$ & $27.455 \pm 24.32$ & Yes & yes \\
\hline
\end{tabular}




\section{Gleason Score}

\section{ET-1 ELISA}

ET-1 ELISA was performed according to the manufacturer's protocol (Fine Test, Wuhan Biological Technology Co. Ltd). 100 $\mu \mathrm{l}$ patients' serum was used for ELISA. Control serum came from patients without urological tumours. Serum from patients belonging to one of the mentioned patient's cohort group was used for the performance of the ELISA.

\section{Vim3 ELISA}

Vim3 ELISA plates were washed 2x with 1xPBS and incubated with 3B4 Vimentin antibody (against full length and truncated version) $1: 500$ for $1 \mathrm{~h}$ at room temperature. Afterwards, wells were washed $2 \mathrm{x}$ in PBS and incubated with patient serums or Vim3 peptide (for calibration) at room temperature for $1 \mathrm{~h}$. Wells were washed again with PBS 3x and incubated with Vim3 antibody overnight at $4^{\circ} \mathrm{C}$. ELISA was washed again with PBS and the plate was incubated with the mouse-HRP labelled secondary antibody for 1 hour at room temperature. TMB was used according to the manufacturer's protocol and all reactions were stopped after exactly $10 \mathrm{~min}$ with stopping solution. For ELISA analysis the FlouoStar Omega reader was used.

\section{Western Blot}

Total protein and Western blots were performed as already published [8]. $25 \mu \mathrm{g}$ of total protein was used for SDS-PAGE loading. All blots were done in triplicates and analysed with the INTAS
Chemostar.

\section{Immunohistochemistry}

$4 \mu \mathrm{m}$ thick paraffin embedded tissue sections from prostatectomy specimens $(n=10)$ were deparaffinised by incubation for $2 \mathrm{x}$ 5 min in Xylene, followed by $2 \times 3$ min 100\% ethanol, and 1 min $95 \%$ ethanol, then rinsed with distilled water. The slides were incubated with a specific serum blocker (anti-rabbit) for $30 \mathrm{~min}$, to avoid unspecific binding. After incubation period, the slides were re-incubated for 1 hour at roomtemperature with specific primary antibodies (Vim3 and VimFL). After washing with PBS-Tween 20, sections were incubated with a secondary anti-rabbit antibody (Santa Cruz). Posteriorly, slides were rinsed with PBS-Tween 20 and washed for $2 \mathrm{~min}$ in 95\% ethanol followed by $2 \times 3 \mathrm{~min}$ in $100 \%$ methanol, lastly, counterstained with hematoxylin and eosin, then coverslipped. All cases were evaluated according to the guidelines [9] using antibodies to the following proteins: AMACR, androgen receptor, p63, PSA, ki67. The Vim3, staining was divided into three categories: 0 (weak or no staining), + (moderate staining), and ++ (strong staining).

\section{Statistics}

All experiments were performed in triplicates. The statistical analysis was done with the GraphPrism 5 (Graphpad software, La Jolla, USA). A paired T-test with one-way ANOVA was performed. The alpha-value was 0.05 and the results are visualized as boxplots $\left({ }^{* * *} \mathrm{p}<0.0001,{ }^{* *} \mathrm{p}<0.001,{ }^{*} \mathrm{p}<0.01\right)$.

\section{Results}

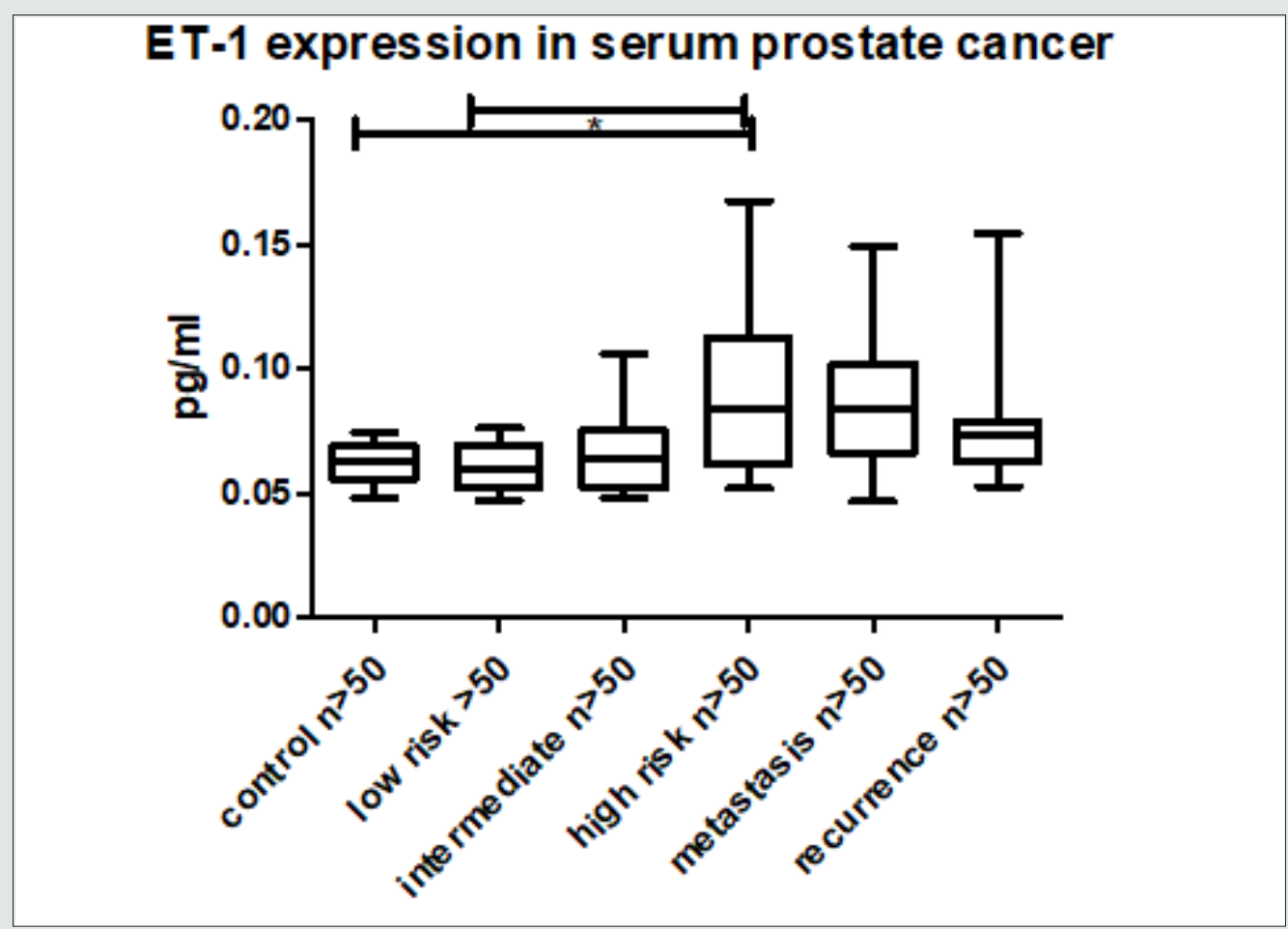

Figure 1: Patient serum ET-1 ELISA. Shows significant higher serum concentration in patients with PCA. 


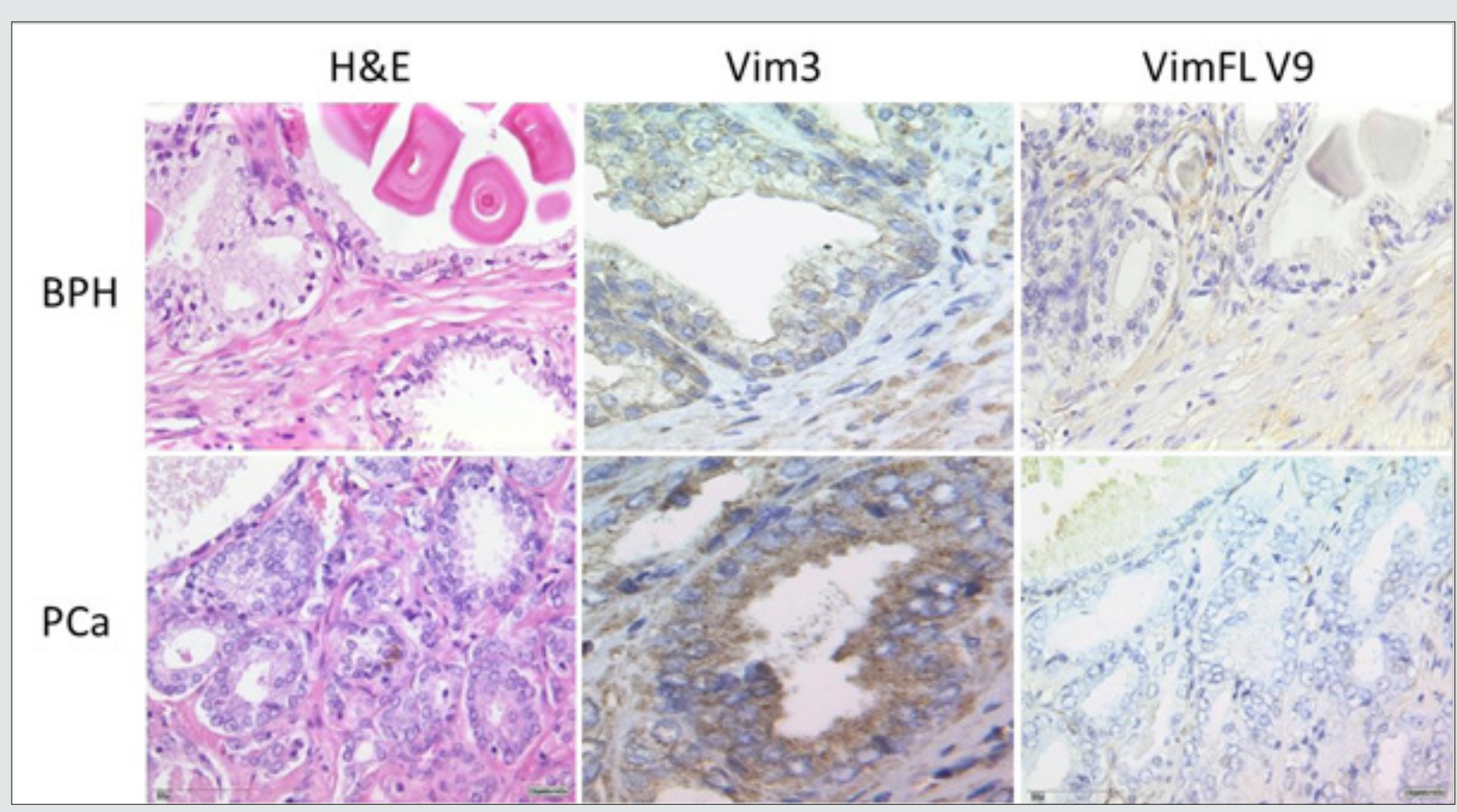

Figure 2: Immunhistochemistry of VimFL and Vim3 in BPH and prostate cancer tissue. Vim3 staining in PCa is stronger. VimFL is negative in PCa and slightly positive in $\mathrm{BPH}$.

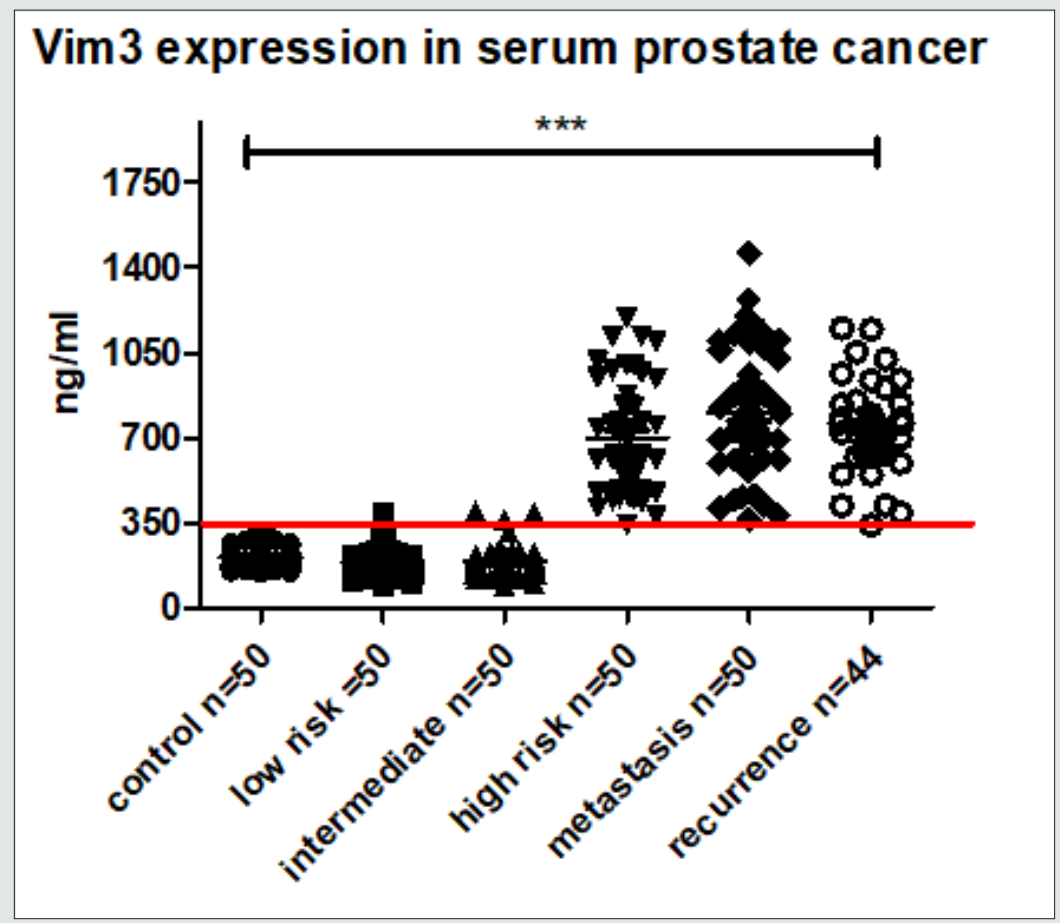

Figure 3: Serum ELISA Vim3. Increasing Vim3 levels were detectable in patients with increasing prostate cancer aggressiveness. "grey-zone" between 300-350 $\mathrm{ng} / \mathrm{ml}$.

Evaluation of ET-1 concentration in PCa patients' serum to identify a possible use of ET-1 level determination as a surrogate biomarker. ET-1 expression in serum was significantly increased $(\mathrm{p}<0.01)$ in patients with high-risk prostate cancer (Figure 1).
Surprisingly, the expression among other groups shows a trend but not significant. In accordance with previous data, a parallel rise in the levels of Vim3 was expect, therefore, serum levels of this Vimentin truncated variant were analyzed in different cohorts. 
Vimentin is used routinely as a marker in immunohistochemistry; therefore, paraffin embedded tissue samples were stained for both types of Vimentin (VimFL and Vim3). The staining of the full-length variant of Vimentin (VimFL) presented nearly the same picture in the benign prostate hyperplasia (BPH) and in prostate cancer. It can be seen that both samples are slightly positive based on the presence of full-length Vimentin (Figure 2). Vim3 expression presents different results. In BPH Vim3 staining was slightly positive $(+)$ whereas staining of the prostate cancer samples shows a stronger, diffuse cytoplasmic positivity (++). In Figure 2, the different intensities of the Vim3 staining pattern can be seen. In case of a high-grade prostate cancer (Gleason 9) the Vim3 expression is stronger in comparison to the BPH. The analysis of the results of Vim3 ELISA serum samples of 314 patients taken from the local biobank after dividing patient samples into different groups, depending on the staging of the previously diagnosed prostate cancer is shown in figure 3. As a control, serum samples from 50 men without cancer history were used. It can be observed that the Vim3 protein level increased in the high risk, metastasis and recurrency group of patients in a very similar fashion. In contrast, the values remained similarly low among the patients with low or intermediate risk PCa's \{Gleason scores 6 and $7(3+4)$ $(4+3)\}$ exhibiting practically no difference in comparison to the control group. A "grey zone" level from which no group can be $100 \%$ demarcated can be observed between 300-350 ng/ml. To further evaluate if the measurement of the Vim3 levels is useful as prognosis marker for treatment success, we divided the high-risk patient group in patients which were successfully treated with androgen deprivation therapy and those which were resistant. As depicted in (Figure 4) a significant decrease was detectable in the group of patients where the androgen deprivation therapy was successful (castration responders).

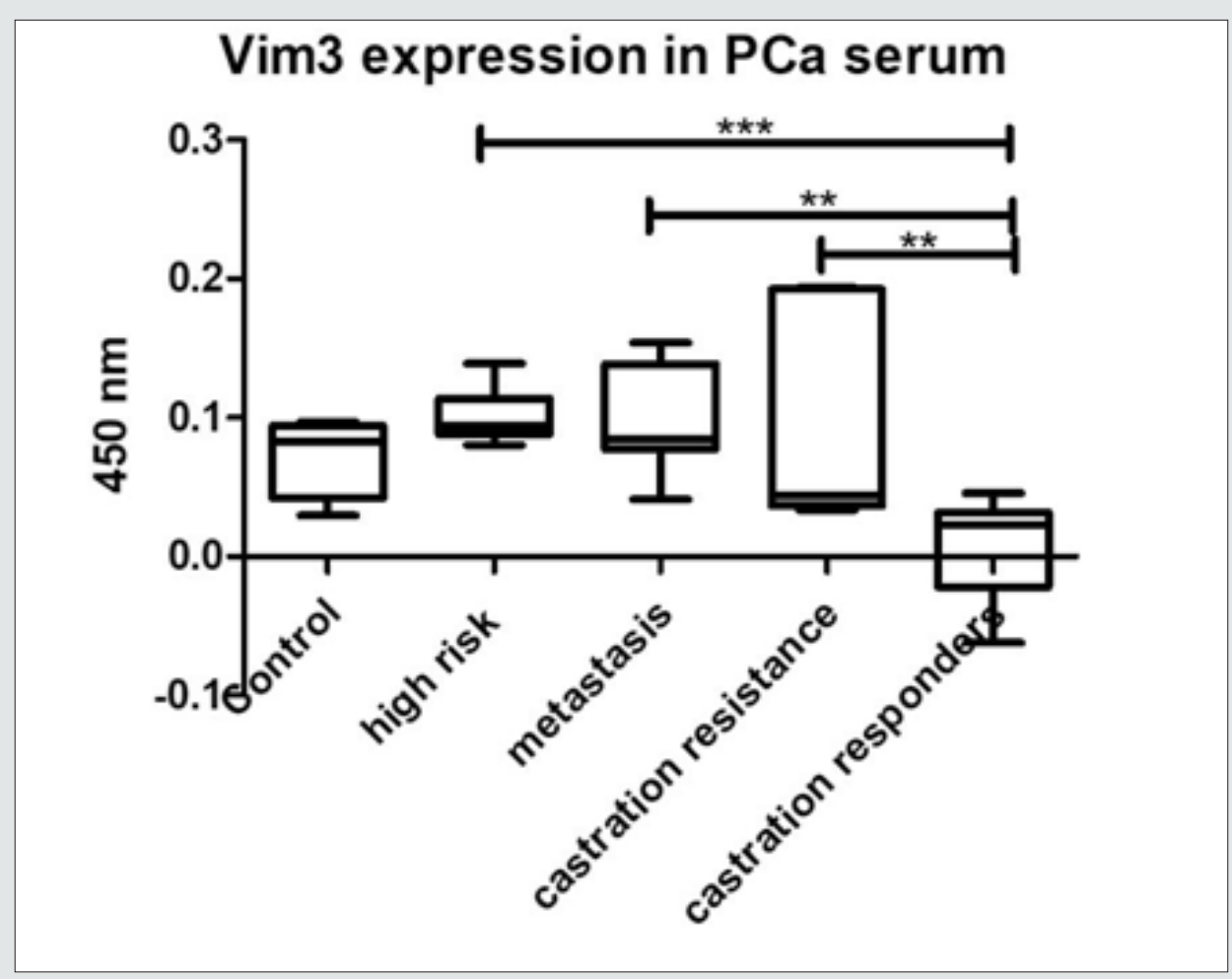

Figure 4: Vim3 Serum ELISA. Decreasing Vim3 detectable in castration responders.

\section{Discussion}

As recently described, ET-1 plays a role in the expression of Vim3 [10] which turn seems to stimulates a highest migration rate of PCa cells in culture when treated with a combination of ET+ETBR blocker according to Koeditz, et al. 2020 [11]. Such effect could be explained by the fact that Vimentin is associated with increase malignancy of cells and is highly expressed in poorly differentiated prostate cancers [12]. Vim3 may possibly be responsible for increasing the metastatic behaviour of cancer cells. However, one issue that apart us from a consensus is the fact that the differentiation of the full-length and truncated Vimentin is quite difficult as available antibodiesdetect either only the full-length variant or both types [13]. Nevertheless, the newly designed Vim3 monoclonal antibody is specific for the truncated version [13]. The results presented in this study are consistent with previous reports of ET-1 overexpression in prostate cancer patients [14,15], as it can be seen that in our Cologne collective, ET-1 levels also increased significantly in comparison with the control group. Nevertheless, the differentiation between the different groups according to the ET-1 expression is not as impressive as it is in the case of Vim3. In control patients, the level of ET-1 was also detectable, since it is also secreted by healthy cells and has different physiological functions [16]. Therefore, measurement of serum Vim3 levels has the potential to be used as a surrogate marker. Vim3 levels in serum samples from PCa patients could represent a useful new surrogate marker for classifying prostate cancer according to tumor aggressiveness 
and possibly also for monitoring or prognosis the response to ADT. Vim3 expression can also be detected by immunohistochemistry and could be used as a marker for prostate cancer with increasing metastatic potential. Nevertheless, our aim is to find a non-invasive biomarker; therefore, a Vim3 ELISA from patients' serum was performed. This ELISA shows that the analysis of Vim3 can be used as a differentiation marker between high risk, intermediate, and low risk prostate cancers. With respect to the grey, we assume that Patients results in this area, even when they were diagnosed as low risk, need to be frequently controlled since, in our opinion, these are the patients who can possibly have a recurrence of the PCa or a metastasis. Nevertheless, this needs to be evaluated and compared to other novel targets for diagnosis of PCa in the future $[17,18]$. Furthermore, we divided the group of patients with high risk PCa further to get an overview if the measurement of Vim3 could be also useful as prognostic marker for response to therapy. For this, serum was analyzed from PCa patients obtained previous to any kind of therapy, with metastases at first contact (without therapy), ADT non-responders (resistant to castration) and ADT responders. Here, depicted in Figure 4, a significant decrease in Vim3 levels was measured in the responders group compared to all other groups.

\section{Conclusions}

Based on published in vitro and in vivo data, we are convinced, that Vim3 might represent a novel surrogate marker which can be used for the grading of prostate cancer patients according to the aggressiveness of the tumour. The Vim3 levels correspond to the Gleason Score. The analysis of Vim3 can be used in combination with the prostate specific antigen (PSA) and the analysis of the Gleason Score to find a personalised therapy option for patients with prostate cancer. Whether patients in the intermediate and high-risk group with and without relapse have different Vim3 levels, needs to be evaluated in further experiments. In prospective studies the Vim3 serum levels should be determined before biopsy to see a correlation between the Gleason Score and the Vim3 level. It is also evident that the results presented here need to be validated in an external cohort in addition to refining the cohorts of the patients analysed and thereby giving more strength to the results Furthermore, even if the group of patients used for the last experiment was smaller, it could be assumed that the measurement of Vim3 via ELISA can be used as treatment prognostic marker.

\section{References}

1. Grant K, M Loizidou, I Taylor (2003) Endothelin-1: a multifunctional molecule in cancer. Br J Cancer 88(2): 163-166.
2. Knowles J, M Loizidou, I Taylor (2005) Endothelin-1 and angiogenesis in cancer. Curr Vasc Pharmacol 3(4): 309-314.

3. Rosano L, F Spinella, A Bagnato (2013) Endothelin 1 in cancer: biological implications and therapeutic opportunities. Nat Rev Cancer 13(9): 637651.

4. Lowery J (2015) Intermediate Filaments Play a Pivotal Role in Regulating Cell Architecture and Function. J Biol Chem 290(28): 17145-17153.

5. Said MV (2017) PSA screening for prostate cancer. Rev Assoc Med Bras 63(8): p. 722-725.

6. Prensner JR (2012) Beyond PSA: the next generation of prostate cancer biomarkers. Sci Transl Med 4(127): 127.

7. Xu N, Yu Peng Wu, Xiao Dong Li, Min Yi Lin, Qing Shui Zheng, et al. (2018) Risk of upgrading from prostate biopsy to radical prostatectomy pathology: Is magnetic resonance imaging-guided biopsy more accurate? J Cancer 9(19): p. 3634-3639.

8. von Brandenstein MG, Anna Ngum Abety, Reinhard Depping, Tanja Roth, Matthias Koehler, Hans-Peter Dienes, et al. (2008) A p38-p65 transcription complex induced by endothelin-1 mediates signal transduction in cancer cells. Biochim Biophys Acta 1783(9): 1613-1622.

9. Humphrey PA (2017) Histopathology of Prostate Cancer. Cold Spring Harb Perspect Med 7(10).

10. von Brandenstein M, Stephan HB, Andreas P, Claudia R, Tobias K, et al. (2018) Beyond the 3'UTR binding-microRNA-induced protein truncation via DNA binding. Oncotarget 9(67): 32855-32867.

11. B KO, Andreas S, Heike G, Isabell H, Jochen F, et al. () Vimentin 3 Expression in Prostate Cancer Cells. Anticancer Res 41(1): 169-174.

12. Satelli A, S Li (2011) Vimentin in cancer and its potential as a molecular target for cancer therapy. Cell Mol Life Sci 68(18): 3033-46.

13. von Brandenstein M, Katharina P, Monika S, Heike L, Joachim PK, et al. (2015) Vimentin 3, the new hope, differentiating RCC versus oncocytoma. Dis Markers 2015: 368534.

14. Weydert CJ, Alison KE, Ruth AM, Justin MD, J Matthew B, et al. (2009) Endothelin-1 inhibits prostate cancer growth in vivo through vasoconstriction of tumor-feeding arterioles. Cancer Biol Ther 8(8): p. 720-729.

15. Nelson JB, K Chan Tack, SP Hedican, SR Magnuson, TJ Opgenorth, et al. (1996) Endothelin-1 production and decreased endothelin B receptor expression in advanced prostate cancer. Cancer Res 56(4): p. 663-668.

16. Guruli G, Beth RP, Stefana P, Valeria M, Michael RS, et al. (2004) Function and survival of dendritic cells depend on endothelin- 1 and endothelin receptor autocrine loops. Blood 104(7): 2107-2115.

17. Cheaito KA, Hisham FB, Ola H, Eman S, Christelle D, al. (2019) EMT Markers in Locally-Advanced Prostate Cancer: Predicting Recurrence? Front Oncol 9: p. 131.

18. Zhang Y, Jiong Z, Suyu L, Genqiang L, Gang L, et al. (2019) Long noncoding RNA VIM-AS1 promotes prostate cancer growth and invasion by regulating epithelial-mesenchymal transition. J BUON 24(5): 20902098. 
(C) (P) This work is licensed under Creative To Submit Your Article Click Here: Submit Article

DOI: $10.32474 /$ OAJOM.2021.04.000189

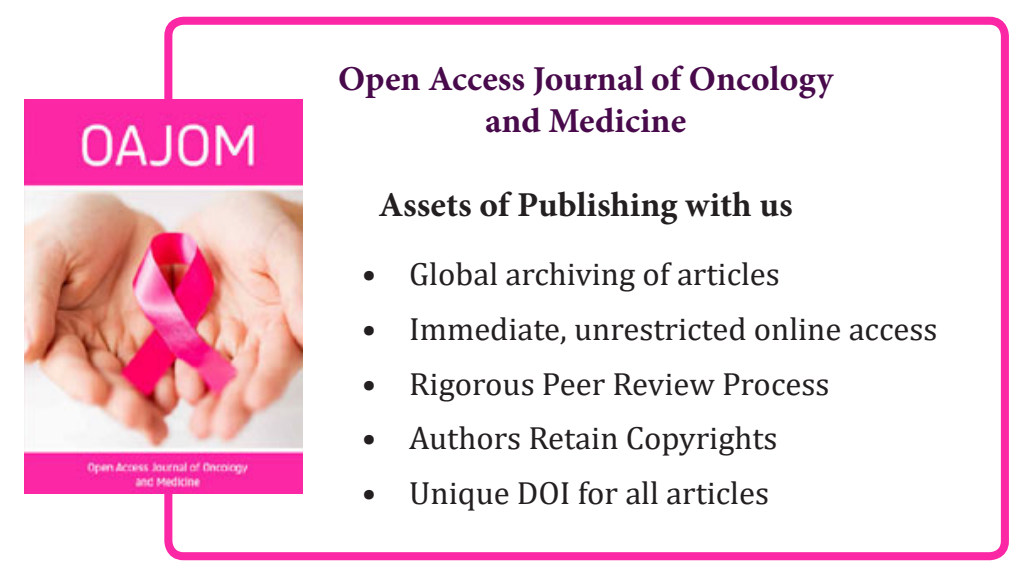

